\title{
30 YEARS OF HIGH-INTENSITY NEGATIVE ION SOURCES FOR ACCELERATORS
}

\author{
Vadim Dudnikov \\ Fermi National Accelerator Laboratory", Batavia, IL 60510, US
}

\begin{abstract}
Thirty years ago, July 1, 1971, significant enhancement of negative ion emission from a gas discharge following an admixture of cesium was observed for the first time. This observation become the basis for the development of Surface Plasma Sources (SPS) for efficient production of negative ions from the interaction of plasma particles with electrodes on which adsorbed cesium reduced the surface work-function. The emission current density of negative ions increased rapidly from $\mathrm{j} \sim 10 \mathrm{~mA} / \mathrm{cm}^{2}$ to $3.7 \mathrm{~A} / \mathrm{cm}^{2}$ with a flat cathode and up to $8 \mathrm{~A} / \mathrm{cm}^{2}$ with an optimized geometrical focusing in the long pulse SPS, and to 0.3 $\mathrm{A} / \mathrm{cm}^{2}$ for DC SPS, recently increased up to $0.7 \mathrm{~A} / \mathrm{cm}^{2}$. Discovery of charge-exchange cooling helped decrease the negative ion temperature $\mathrm{T}$ below $1 \mathrm{eV}$, and increase brightness by many orders to a level compatible with the best proton sources, $B=j / T>1 \mathrm{~A} / \mathrm{cm}^{2} \mathrm{eV}$. The combination of the SPS with charge-exchange injection improved large accelerators operation and has permitted beam accumulation up to space-charge limit and overcome this limit several times. The early SPS for accelerators have been in operation without modification for $\sim 25$ years. Advanced version of the SPS for accelerators will be described. Features of negative ion beam formation, transportation, space-charge neutralization- overneutralization, and instability damping will be considered. Practical aspects of SPS operation and high brightness beam production will be discussed.
\end{abstract}

\section{INTRODUCTION}

One practical result of development of high brightness negative ion source is accepting of the charge-exchange injection in circular accelerators for a routine operation. Now negative ion sources are "Sources of life" for gigantic accelerators complexes as FNAL, BNL, KEK,.. and an efficiency and reliability of these sources operation are determined a productivity of these big collaborations. Many results of the high energy physics were discovered with using of negative ion sources. Development of high brightness $\mathrm{H}^{-}$sources was stimulated by first success of high current proton beam accumulation with using a charge-exchange injection [1] and supported by interest of "Star War" [2]. A recent circumference was a reason of difficulties and long delay of publications, but nonofficial communication was relative fast. Until 1971 a main attention was concentrated on the charge-exchange ion sources, because was no hope to extract from the plasma directly more than $5 \mathrm{~mA}$ of $\mathrm{H}^{-}$. At July 1, 1971 at the Institute of Nuclear Physic (INP), Novosibirsk, by author almost occasionally was observed very short in time enhancement of negative ion emission from the magnetron (planotron) plasma source following by introducing a cesium admixture to the gas discharge. Fortunately this brief observation, considered in review [3], was not lost and was developed and understood as a new method of negative ion production in interaction of plasma with a surface and a basis for development of a Surface Plasma Sources (SPS). In the patent application [4] was considered " a method of negative ion production in gas discharges, distinguished by adding to the discharge along with a working substance an admixture of substance with a low ionization potential as cesium, for example, for enhance a negative ion formation". Further development of SPS was conducted by cooperation Belchenko, Dimov, Dudnikov (BDD). The first high brightness SPS for accelerator has been developed by author [5]. The Semiplanatron SPS with an efficient geometrical focusing has been developed by author at 1977 [6]. Further R\&D of high brightness SPS was conducted in cooperation with Derevyankin. Very fast development and adaptation of SPS has been start in many USA laboratories, in Europe and in Japan, and International Symposiums for Production and Neutralization of Negative Ions and Beams has been established [7]. Now the Surface Plasma Method of negative ion production and SPS considered in many books (recently in book [8]). Good review of SPS for accelerators presented in reports of J. Peters [9-11]. Development of high current SPS (tens of Ams) for thermonuclear plasma heating is in progress and used in experiments [12]. Production of polarized negative ions by charge- exchange with a slow negative ion in SPS has been proposed by author and has been realized with a good success [13]. This development has permeate to use a charge exchange injection for accumulation of high intense beams of polarized ions in circular accelerators. In R\&D of heavy negative ion SPS for technology application a good success has been reached [14].

\section{FEATURES OF SPS}

The efficiency of negative ion formation depends very much on the catalytic property of the surface, mainly the work-function. For enhanced negative ion formation in 
the SPS a mixture of substances with a low ionization energy, such as alkaline or alkaline earth elements or compounds, are used. Most efficient is the addition of cesium. Still the surface work-function and catalytic properties of the surface for negative ion formation depends very much on many parameters such as surfacecesium concentration, admixtures of other compounds, such as oxides, halides, nitrides, and surface temperature. Small changes in the surface condition dramatically change the efficiency of negative ion formation. It is a fine art and some magic to optimize the surface and plasma condition for high efficiency of negative ion formation. This condition is a strong reason for the variation in efficiency of negative ion production although conditions look very similar. Small changes in the surface condition can increase or decrease the intensity of a negative ion beam by large factors. Often the intensity of $\mathrm{H}^{-}$and $\mathrm{D}^{-}$beams from a $1 \times 10 \mathrm{~mm}$ emission slit as a function of discharge current $I_{d}$ could vary from $200 \mathrm{~mA}$ to $10 \mathrm{~mA}$ for the same discharge current. A stronger variation can has a beam brightness. An efficient ion temperature can have a variations from a

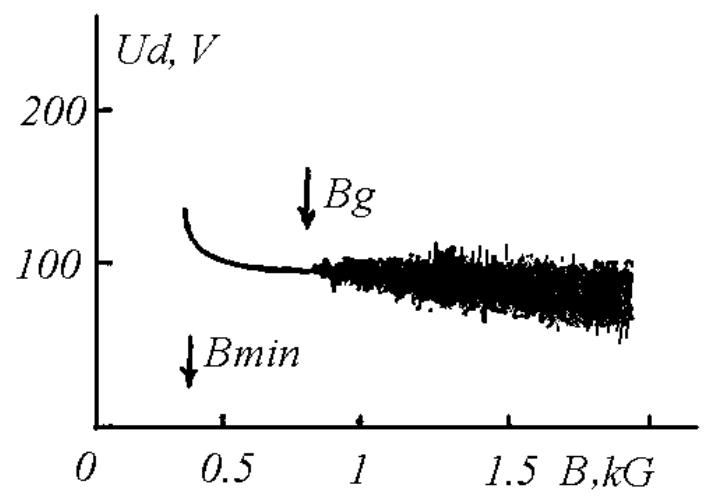

Figure 1: The discharge voltage and level of noise vs. magnetic field in SPS with Penning geometry.

part of $\mathrm{eV}$ to some $\mathrm{keV}$. It is easier to have stable operation with relatively low beam parameters such as intensity I $\sim 30-50 \mathrm{~mA}$, emission current density $\mathrm{J} \sim 0.5-1$ $\mathrm{A} / \mathrm{cm}^{2}$, transverse ion temperature $\mathrm{Ti} \sim 5-10 \mathrm{eV}$. Present experience permits better optimization for long stable production of high-brightness high-intensity beams of negative ions $\left(\mathrm{I} \sim 0.1-0.15 \mathrm{~A}, \mathrm{~B} \sim \mathrm{J} / \mathrm{Ti}>1 \mathrm{~A} / \mathrm{cm}^{2} \mathrm{eV}\right.$, lifetime $\mathrm{N}>10^{8}-10^{9}$ pulses). Highest brightness could be reached only with noiseless operation. The level of discharge noise (hash) is depend of many parameters. For stable discharge a surface properties should be in the stable conditions and frequency of electron scattering by plasma particles should be higher than Larmour frequency. A discharge noise could be suppressed by decrease of magnetic field as shown in Fig. 1 and by increase a gas or Cs density. Admixture of heavy gas could be useful for noise suppression, but it increase a sputtering. Examples of discharge voltages with a different level of noise are shown in Fig. 2. A transition from the noise discharge $(a)$ to noiseless one $(c)$ increases a beam brightness at order of magnitude.

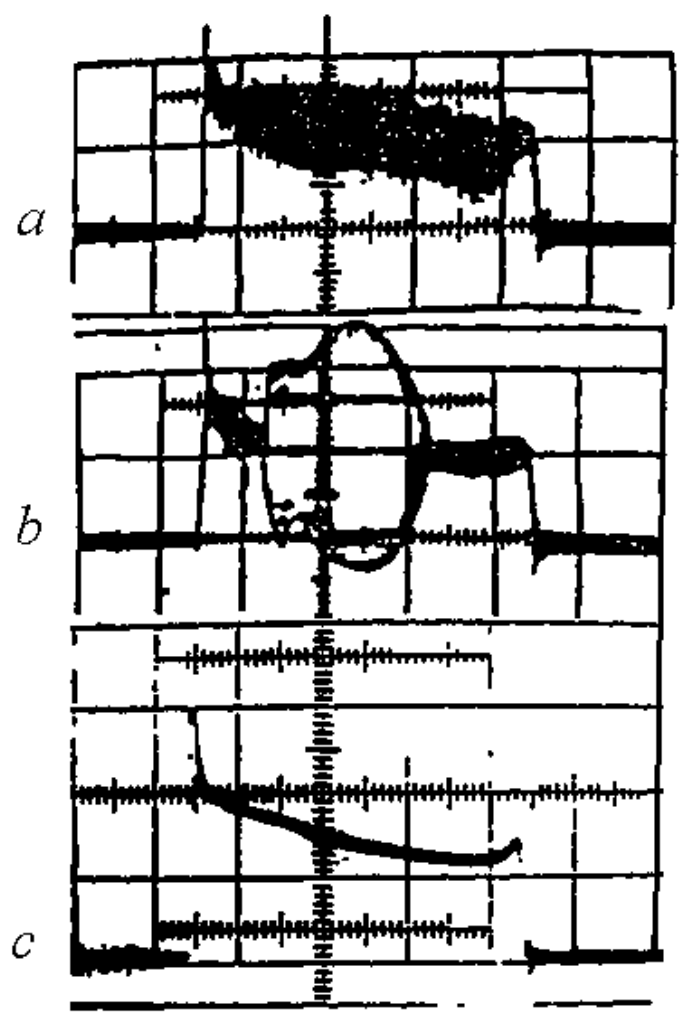

Figure 2: The examples of discharge voltages for different conditions in SPS. (a) a discharge with noise; $(b)$ a discharge with RF generation: (c) noiseless discharge.

Vertical scale is $100 \mathrm{~V} /$ div; Horizontal scale is $0.2 \mathrm{~ms} / \mathrm{div}$.

\section{NEGATIVE ION SOURCES FOR ACELERATORS}

The first versions of the Surface-Plasma Sources (SPS) developed for charge-exchange injection of protons have an operating intensity I $\sim 50 \mathrm{~mA}$ with pulse lengths of 0.05-1 msec, noisy discharges and a repetition rate up to $50 \mathrm{~Hz}$ [6-9]. $\mathrm{H}^{-}$beam parameters of these SPS was sufficient for normal operation of large proton accelerator complexes during the past 25 years without significant modernization of ion sources. Now, new accelerator projects need an increase of the ion beam intensity and brightness. Some upgrading of existing SPS could achieve the necessary increase of intensity, duty factor and beam quality without degradation of reliability and availability of the achieved satisfaction level.

The Fermilab Magnetron SPS has been operational since 1978 [15]. The peak current of the $\mathrm{H}^{-}$ion beam at the exit of the $750 \mathrm{keV}$ accelerator column is $\mathrm{I}_{\mathrm{b}}=65 \mathrm{~mA}$ with an extraction voltage $\mathrm{Uex}=20 \mathrm{kV}$, and $\mathrm{I}_{\mathrm{b}} \sim 70 \mathrm{~mA}$ with Uex $=25 \mathrm{kV}$ with a beam pulse length $\mathrm{T}=0.075 \mathrm{msec}$ at 15 Hz. The pulse length could be increased with a new arc discharge pulser and adjusted parameters. It is useful for stable operation to have a discharge power supply as a current source with a high impedance $(Z=5-10$ Ohm, now $\mathrm{Z}=1 \mathrm{Ohm}$ ) and corresponding higher voltage. Optimization of the discharge electrode configuration 
should help to increase the intensity above $\mathrm{I}_{\mathrm{b}}=0.1 \mathrm{~A}$ without increasing the discharge power above acceptable levels. Gas delivery optimization should allow a longer pulse and higher intensity without an increase of the gas loading.

An optimized extraction system with a suppression electrode should improve the beam intensity, beam quality and beam space-charge neutralization with a low gas pressure. A suppression of the positive ion extraction to the accelerating gap should suppress cathode and anode sputtering by accelerated positive ions - a main reason for the short ion source lifetime. Improved cathode and anode cooling is necessary for increased discharge pulse length and intensity. The Semiplanotron version of the SPS is good for operation at higher duty factor.

From previous experience it is possible to have reliable operation of a SPS with parameters: peak current after extraction (bending magnet) $\mathrm{I}_{\mathrm{b}} \sim 0.12-0.15$ A with pulse duration of $\mathrm{T} \sim 0.25 \mathrm{msec}$, repetition rate $\mathrm{F}=100 \mathrm{~Hz}$ normalized emittance $\varepsilon(90 \%)=1 \pi \mathrm{mm}$ mrad. SPS with these parameters was tested with a relatively long run [16].

Lifetime of SPS determined by electrode sputtering and flakes formation. It is dominate a cathode or anode sputtering by back accelerated positive ions. Suppression of positive ion is important for increase lifetime.

Optimized cesium film recycling (deposition-desorbtion) could be used for shielding of electrodes from the sputtering and can reduce the sputtering to a very low level. Cesium in the SPS acts as an oil in an engine, increasing the operational lifetime. "Cold Start" of a discharge without cesium for a few minutes could be more destructive than many hours of low voltage operation. Emission current density of $\mathrm{H}^{-}$up to $\mathrm{J} \sim 1$ $\mathrm{A} / \mathrm{cm}^{2}$ has been observed in discharges without cesium. A fingerprint with a trace of $\mathrm{Na}$ or $\mathrm{K}$ could increase the efficiency of $\mathrm{H}^{-}$production significantly. But the power density in a discharge without cesium is very high and the sputtering rate is much higher. Electron emission from ion source without cesium is very high.

Recently, new version of RF SPS has been developed in DESY[9-11], SPS with pulsed arc discharge in Frankfurt University and in KEK for high intense Proton Driver.

\section{LOW ENERGY BEAM TRANSPORT}

The ion beam from a compact SPS has a very high current density $\left(\mathrm{j} \sim 1-3 \mathrm{~A} / \mathrm{cm}^{2}\right)$ and perveance. For transport of these beams it is necessary to use a deep space-charge neutralization (compensation) or very strong continuous focusing by electrostatic forces as in the RFQ.

Partial compensation of space charge with magnetic focusing and nosy operation will create a strong variation of focusing and lead to an increase of emittance by ellipse rotation. Still, this mode of transport is used in almost all injectors, and until recently it was acceptable. Space charge compensation by ions has some difference from the compensation by electrons. Ion oscillation in the potential of the beam is more coherent and can be a reason for very strong and fast beam-ion instability.
Beam-ion instabilities have been observed recently in the electron beam of the Advanced Light Source (LBL) with increased residual gas density. In low energy negative ion beams this instability has been observed many years ago (1976). A development of this instability along $\mathrm{H}^{-}$beam, coherent oscillations of positive ions in the beam potential excite quadruple and dipole oscillation of the $\mathrm{H}^{-}$beam, and developed a decompensation and emittance growth.

To eliminate this problem many versions of an electrostatic focusing-transport LEBT has been proposed. Now under development and testing is an ELEBT for SNS. Transport of a $\mathrm{H}^{-}$beam of energy $65 \mathrm{keV}$ with intensity up to $40 \mathrm{~mA}$ under development [16].

The beam-ion instability could be damped by overneutralization of the beam, changing the sign of the beam potential with an increase of the ion density in the beam. With increased an ion and electron density a stable beam transport could be reached with additional focusing by reversed space charge. This solution could be used for a short transport with acceptable levels of ion loss by stripping. This solution is convenient because it is possible to locate a second (spare) ion source in front of one RFQ. Ion beam pulses from this ion source could be long enough for reaching a deep over-neutralization.

A good solution could be a short LEBT with a fast beam over-neutralization by streams of noiseless plasma from a separate plasma source. With magnetic focusing, beams from 2 SPS could be steered to the entry of the RFQ. Close-coupled systems has been tested in ion implantation.

\section{REFERENCES}

[1] G.Budker, G. Dimov, V. Dudnikov, in Proc. Internat. Symposium on Electron and Positron Storage Ring, France, Sakley,1966, rep. VIII, 6.1 (1966)

[2] C. Robinson, Aviation Week\&Space Tech.,p.42, oct., 1978. Rev. Mod. Phys., 59(3), Part II, 1987.

[3] V. Dudnikov, Rev. Sci. Instrum., 63(4),2660 (1992).

[4] V.Dudnikov, The Method for Negative Ion Production, SU patent, C1.H013/04, No 411542, Appl. 3/3/72.

[5] V. Dudnikov, Proc. $4^{\text {th }}$ All-Union Conf. On Charged Part. Accel.,Moscow,1974' V.1, p.323.

[6] V. Dudnikov, Yu. Belchenko, Preprint, INP 78-95, Novosibirsk,1978.

[7] C.W. Schmidt, C. Curtis, IEEE Trans. Nucl. Sci. NS26,4120 (1979).

[8] H. Zhang, Ion Sources, Springer,1999

[9] J.Peters, LINAC' 98, Chicago, 1998.

[10] J.Peters, Rev. Sci. Instrum., 71(2),1069 (2000).

[11] J.Peters, EPAC'2000.

[12] Y.Okumura, et al., Rev. Sci. Instrum.,71(2),1219 (2000).

[13] A. Belov, V. Derinchuk, This Conference.

[14] J. Ishikawa, Rev.Sci.Instrum., 67(3) 1410 (1996).

[15] C. W. Schmidt, Prod. Neutralizat. of Negative Ions and Beams, $8^{\text {th }}$ Internat. Symp. AIP 1-56396-773-5.1998.

[16] G. Dimov, V. Dudnikov, G. Derevyankin, IEEE Trans. Nucl. Sci. NS-24,1545 (1977).

[17] R. Keller et al., This conference. 